$\begin{array}{ll}\text { Revue d'Alsace } & \text { Revue d'Alsace } \\ & 133 \mid 2007 \\ & \text { Histoire régionale Landesgeschichte en France et en } \\ & \text { Allemagne } 1950 / 2000\end{array}$

\title{
Chronique des Archives
}

Jean-Luc Eichenlaub

\section{(2) OpenEdition}

12 Journals

Édition électronique

URL : http://journals.openedition.org/alsace/1505

DOI : $10.4000 /$ alsace. 1505

ISSN : 2260-2941

Éditeur

Fédération des Sociétés d'Histoire et d'Archéologie d'Alsace

Édition imprimée

Date de publication : 1 octobre 2007

Pagination : 501-504

ISSN : 0181-0448

\section{Référence électronique}

Jean-Luc Eichenlaub, «Chronique des Archives », Revue d'Alsace [En ligne], 133 | 2007, mis en ligne le

10 novembre 2011, consulté le 30 avril 2019. URL : http://journals.openedition.org/alsace/1505 ; DOI : 10.4000 /alsace. 1505 
Jean-Luc EICHENLAUB

\section{Chronique des Archives}

Ces quelques lignes n'ont d'autre ambition que de donner un état de ce qui se fait dans les différents services d'archives de la région qui ont bien voulu répondre, et que je remercie ici (pour les archives départementales du Bas-Rhin Pascale Verdier, pour Erstein Vincent Husser, pour Haguenau Michel Traband, pour Sélestat Hubert Meyer, pour Strasbourg Laurence Perry, pour Colmar Francis Lichtlé, pour Guebwiller Sophie Coignot-Genin, pour Illzach Agathe Antony, pour Saint-Louis Sylvie Meyer, pour Soultzmatt Céline Frank, pour Turckheim Florent Edel). Indiquant les principales entrées, elles constituent aussi une source précieuse sur la localisation de fonds qui ne seront accessibles qu' une fois classés. Le chercheur consultera avec intérêt les différents sites Internet existant où il pourra trouver instruments de recherche (répertoires, inventaires, bordereaux de versement par exemple) et parfois documents en ligne. Il pourra ainsi au moins préparer ses visites voire faire certaines de ses recherches chez lui.

\section{Archives départementales du Bas-Rhin}

Accroissement de plus de 100 mètres linéaires, notamment des archives notariales anciennes. Entrée d'un manuscrit sur l'histoire des Juifs en Alsace (1809-1818), de deux terriers du chapitre de Saint-Pierre-le-Jeune de Strasbourg à Bernolsheim et Wahlenheim (1723).

Des visas d'élimination ont été accordés pour plus de 9000 mètres linéaires d'archives publiques.

De nombreuses réunions sur l'archivage des données numériques ont eu lieu.

Les opérations de classement ont principalement porté sur les archives des notaires.

Les 6289 bobines de microfilm du service ont été numérisées ; 3 millions d'images seront bientôt accessibles grâce à un logiciel de visualisation.

Près de $65 \%$ des lecteurs sont des généalogistes. Un peu plus de $11 \%$ sont des chercheurs scientifiques (master, thèse). 


\section{Archives départementales du Haut-Rhin}

Accroissement de près de 700 mètres linéaires, principalement des archives publiques (Justice, Mines de potasse d'Alsace). 36 mètres linéaires d'archives privées, parmi lesquelles il convient de relever des archives de la famille de Reiset, de la famille Waldner de Freundstein, des archives d'entreprise (échantillons Haussmann au Logelbach, Scheurer-Lauth à Thann, archives de la filature et tissage Klein à Linthal), des archives photographiques (Boehrer à Colmar).

Des visas d'élimination ont été accordés pour plus de 4400 mètres linéaires d'archives publiques.

Les administrations étant rentrées dans l'ère de la dématérialisation, les archives départementales ont travaillé avec elles pour préparer au mieux la conservation à long terme des données ; les travaux ont été particulièrement suivis avec la future maison départementale des personnes handicapées, le ministère de la Justice (spécialement le livre foncier), les services fiscaux (cadastre). La journée d'études organisée à Illzach en décembre a porté en particulier sur cette question.

Près de 800 pages d'instruments de recherche ont été réalisées ; elles sont mises en ligne sur le site Internet des archives au fur et à mesure ; sur ce site peuvent également être consultées trois bases de données: plans de finage (vers 1760-1762), listes des maires et photographies des mairies (depuis la Révolution), photographies des monuments aux morts et listes des victimes.

Les travaux de numérisation ont porté sur les documents figurés et sur l'état-civil (1793-1892). La mise en ligne est prévue à partir de 2008.

La fréquentation de la salle de lecture est stable ; les communications se répartissent ainsi : bibliothèque $5 \%$, microfilms (registres paroissiaux et état civil) $40 \%$, archives $55 \%$. Les recherches les plus nombreuses (42\%) ont été menées sur les archives modernes (1790-1870), suivies de près par les archives anciennes (38\%), et de plus loin par les archives contemporaines (postérieures à 1940-1945) 20 \%. Les chercheurs universitaires sont fort peu nombreux.

\section{Archives communales}

\section{Bas-Rhin}

\section{- Erstein}

Accroissement de plus de 5 mètres linéaires d'archives (archives administratives contemporaines) ; des instruments de recherche informatisés sont mis à la disposition des lecteurs.

Les archives ont réalisé une exposition sur les quatre-vingts ans de l'hôtel de ville. 


\section{- Haguenau}

Accroissement de plus de 11 mètres linéaires d'archives ; les travaux informatiques ont été poursuivis : désormais les dépouillements informatisés des registres paroissiaux et d'état civil, de 1605 à 1905, des registres de recensement, à partir de 1802 sont disponibles en salle de lecture. Parallèlement se poursuit une vaste opération de restauration des registres anciens.

\section{- Sélestat}

Les archives anciennes sont conservées à la Bibliothèque humaniste.

Deux expositions ont été réalisées: "A la redécouverte des dominicains de Sélestat ", "La première mention écrite de l'arbre de Noël ».

\section{- Strasbourg}

Près de 340 mètres linéaires d'archives sont entrés en 2006 ; à noter en particulier le versement de l'atelier de costumes de l'Opéra du Rhin, le don des archives du facteur d'orgues Roethinger, le fonds de l'orphelinat du Neudorf.

En matière de numérisation ont été traités les recensements militaires, les registres de population, les annuaires d'adresses; la numérisation du cadastre napoléonien, du fonds de plaques de verre Toni et des plans du Grand Strasbourg nazi a été entreprise.

Deux expositions avec catalogue: "Les relations franco allemandes vues à travers les atlas scolaires français et allemands " (avec le Landesarchiv de Stuttgart et la Bibliothèque municipale de Strasbourg), «Vivre ensemble " à l'occasion du $40^{\mathrm{e}}$ anniversaire du texte fondateur de la CUS.

\section{Haut-Rhin}

\section{- Colmar}

Accroissement de 35 mètres linéaires. L'index thématique du fonds des archives contemporaines (501 mètres linéaires, principalement deuxième moitié du XX siècle) a été achevé. Le travail se poursuit avec le traitement des archives de la période 1871-1940.

La Société d'histoire et d'archéologie de Colmar a son siège aux archives municipales

\section{- Guebwiller}

Accroissement de plus de 8 mètres linéaires; à noter l'entrée des archives de l'amicale du personnel de la ville de Guebwiller, de l'office municipal de coordination des sociétés patriotiques et d'anciens combattants. Plusieurs fonds ont été classés : les archives du CCAS, la collection des délibérations du conseil municipal de 1790 à 1999. Les inventaires sont réalisés grâce au logiciel Avenio. 
La politique de numérisation des actes d'état civil a abouti aux résultats suivants : tout l'état civil (naissance, mariage, décès) de 1841 à nos jours est numérisé ; les actes de 1900 à nos jours sont de plus indexés.

\section{- Illzach}

Un nouveau local d'archives a été aménagé.

Accroissement de 64 mètres linéaires ; classement du fonds de l'association «Les colonies de vacances d'Illzach» (1938-1992).

\section{- Saint-Louis}

Accroissement de près de 45 mètres linéaires ; réalisation, avec le logiciel Avenio, de 145 pages d'inventaire.

Les archives ont été ouvertes lors des Journées européennes du Patrimoine (17 septembre). La société d'histoire de Saint-Louis a rassemblé d'anciennes photos de classe qui ont été présentées aux archives.

\section{- Soultzmatt}

Accroissement de plus de 15 mètres linéaires. A noter l'entrée des archives de Gérard Bihler, et de celles de l'association Rencontres Art et Culture sur l'exposition "Le coq dans tous ses états et en toute liberté », et d'archives de la Régie communale des Sources de Soultzmatt (1952-2000).

Un inventaire des archives contemporaines de la communauté de communes de la Vallée Noble est en cours d'achèvement.

\section{- Turckheim}

Le classement des articles de la presse locale sur Turckheim (1981-2005) est achevé ; un inventaire des œuvres des musiciens Constant et Victor Sieg a été réalisé.

L'archiviste participe à l'élaboration du bulletin communal. 